\title{
Qualification and characterization of electronics of the fast neutron Hodoscope detectors using neutrons from CABRI core
}

\author{
S. Mirotta, IRSN, J. Guillot, IRSN, V. Chevalier, IRSN, B. Biard, IRSN
}

\begin{abstract}
The study of Reactivity Initiated Accidents (RIA) is important to determine up to which limits nuclear fuels can withstand such accidents without clad failure. The CABRI International Program (CIP), conducted by IRSN under an OECD/NEA agreement, has been launched to perform representative RIA Integral Effect Tests (IET) on real irradiated fuel rods in prototypical Pressurized Water Reactors (PWR) conditions. For this purpose, the CABRI experimental pulse reactor, operated by CEA in Cadarache, France, has been strongly renovated, and equipped with a pressurized water loop. The behavior of the test rod, located in that loop in the center of the driver core, is followed in real time during the power transients thanks to the hodoscope, a unique online fuel motion monitoring system, and one of the major distinctive features of CABRI. The hodoscope measures the fast neutrons emitted by the tested rod during the power pulse with a complete set of 153 Fission Chambers and 153 Proton Recoil Counters. During the CABRI facility renovation, the electronic chain of these detectors has been upgraded. In this paper, the performance of the new system is presented describing gain calibration methodology in order to get maximal Signal/Noise ratio for amplification modules, threshold tuning methodology for the discrimination modules (old and new ones), and linear detectors response limit versus different reactor powers for the whole electronic chain.
\end{abstract}

Index Terms - CABRI, Hodoscope, pulse mode, nuclear electronic chain, RIA accident

S. Mirotta, IRSN/PSN-RES/SEREX/L2EP, Cadarache, Saint Paul Lez Durance 13115 FRANCE (email: salvatore.mirotta@irsn.fr),

J. Guillot, IRSN/PSN-RES/SEREX/L2EP, Cadarache, Saint Paul Lez Durance 13115 FRANCE,

V. Chevalier, IRSN/PSN-RES/SEREX/L2EP, Cadarache, Saint Paul Lez Durance 13115 FRANCE,

B. Biard, IRSN/PSN-RES/SEREX/L2EP, Cadarache, Saint Paul Lez Durance 13115 FRANCE

\section{INTRODUCTION}

$\mathrm{O}$ ne of the most challenging problems of our day, in nuclear industry, is to maintain Nuclear Power Plants (NPP) in safe working conditions during all their life. In order to achieve this goal, defense in depth strategy is used to develop nuclear reactor. This strategy consists in some physical barrier between de radioactive materials and the external environment. The first barrier of this methodology is realized by the fuel rod cladding. It is really important that the cladding resists to all reactor's operations (either in normal operating conditions or in severe accident conditions).

Despite all precautions to maintain mechanical conditions for the cladding, in some cases, (i.e. during severe accidents) it may fail. Among the different accidents that could occur in a NPP and could cause clad failure, one of the most important is Reactivity Initiated Accident (RIA). This kind of accident produces a high power excursion in several milliseconds (ms) which may damage cladding and the core geometry. The cladding rupture probability due to a RIA accident depends on several factors (i.e. Zirconia thickness, fuel burnup, high hydrogen content into the cladding, new cladding materials, etc.). CABRI core was built in order to determine up which limits fuel rod cladding does not fail under power excursion due to RIA accident.

Since the beginning, CABRI was equipped by a sodium loop in order to reproduce thermal hydraulic conditions of Sodium Fast Reactors (SFR). In the last period, an intensive renovation of the facility has permitted the replacement of the sodium loop by a pressurized water loop. This new circuit allows experimental studies of RIA in all its phases for Pressurized Water Reactors (PWR).

A test rodlet, refabricated from a rod irradiated in a NPP, is placed into that pressurized water loop, in the center of the core, and is submitted to a short but powerful transient, that may reach $20 \mathrm{GW}$ in some ms. In CABRI, two none destructive systems were built in order to study the behavior of fuel under RIA conditions: 
- A non-destructive examination facility, for performing X-ray radiography and tomography imaging before and after power transient thanks to a linear accelerator;

- An online fuel motion measurement system named hodoscope, which aims at analyzing the fuel motion deduced from the detection of fast neutrons emitted by the tested rod, in real time (with a time step of $1 \mathrm{~ms}$ ) during the transient.

These neutrons emerging from the tested rod are collimated towards a bank of detectors through 153 line-of-sights in a huge steel piece ( $3 \mathrm{~m}$ length and more than 2 ton weight). At the rear face of the collimator, 306 detectors are arranged by pair in a 51 row by 3 column matrix. The collimator is motioned in rotation so as to align the detectors with the test rod.

Two different technologies of detectors are used in CABRI in order to cover the whole power range of the transient, starting at low power $(\sim 100 \mathrm{~kW})$ up to more than $20 \mathrm{GW}$. Proton Recoil Counters (PR) are better suited for low power measurement while ${ }^{237} \mathrm{~Np}$ Fission Chambers (FC) are dedicated to high power monitoring. Both detectors measure the fast neutrons coming from the test rod and from the CABRI driver core fuel. For limiting the neutron contribution due to the CABRI core and maximizing the signal due to the tested rod, the core is divided into two symmetrical half parts by two air channels so that the collimator does not directly see the core fuel. Each detector is associated to a remote electronic chain which role is to shape signals and select interesting events.

Recently, with the collaboration of CEA LIST, these electronic chains have been renewed because of difficulties to maintain old systems. This article will describe the behavior of new amplifier and discriminator compared to those of the old electronic chain. The main goal of this renovation consists in replacing the obsolete integrated components with sustainable discrete components, while maintaining the same characteristics. Although electronics for both PR and FC detectors were created, in this article we will just discuss results obtained for FC detectors because results are similar.

In II a brief description of the electronic chain is presented, in III experimental measures with the amplifier are carried out. In IV, measurements with the discriminator are realized as well. Moreover, in $\mathrm{V}$ the analysis of the entire chain with CABRI neutrons is investigated to determine their real performances. Furthermore, conclusion and perspectives are discussed in section VI.

\section{DETECTOR CHAIN}

All the electronic chains of the hodoscope system are analogic. In Figure II.1, one of the 2x153 measurement chains is described. Detectors are placed behind a collimator $4 \mathrm{~m}$ far from the test rod. A $30 \mathrm{~m} \mu$-metal cable links detector with amplifier. Amplifier and discriminator are connected together, discriminator produce a TTL signal each time impulsions coming from the amplifier pass a threshold value. At the end of the chain a computer converts the TTL signals in counts which are registered in a scaler with a memory of $2^{16}$ bytes. As shown in the figure, the High Voltage (HV) and signals share the same cable.

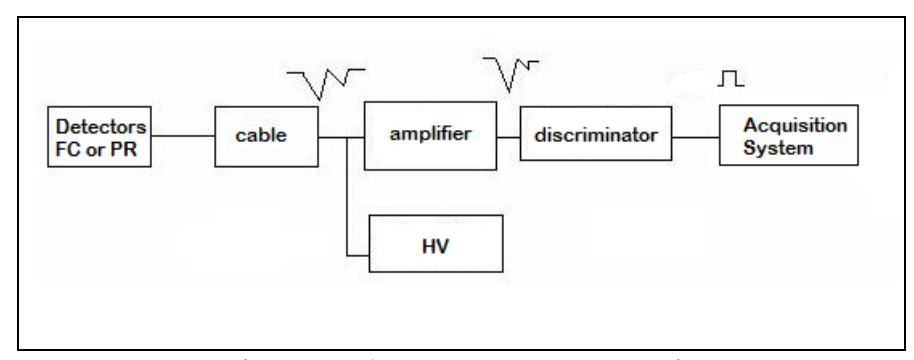

Figure II.1 - Measurement chain

All 306 detectors are used in pulse mode (detectors are placed really far from the core and the fast neutron flux is of the right magnitude for this kind of analysis). Amplifiers and discriminators are placed far $(30 \mathrm{~m})$ from the detectors and they are linked to them by a coaxial cable with double $\mu$-metal shielding. These electronics were recently renewed in order to assess obsolescence of electronic components.

The new electronics was developed in collaboration with CEA LIST laboratories, and they have been tested in CABRI.

Each pair consisting of an amplifier and a discriminator is powered by 3 Direct Current (DC) voltage (12V, 5V and $-5 \mathrm{~V})$. Each DC source supplies 10 measurement chains. Similarly, a HV polarizes 10 detectors simultaneously. All components into the electronic chain are adapted with an impedance of 50 $\Omega$. [1]

\section{AMPLIFIER}

\section{A. Description}

The amplifier is a trans-resistance amplifier, which collects current produced from the particles interacting with gas into the ionization chambers and converts this current in a pulse voltage signal.

This amplifier consists of different stages. As detector signal and HV share the same cable, the input stage comprises a circuit for decoupling and filtering the HV. Moreover, in order to avoid any damage to the following amplification stage, a protection circuit against over current is implemented as well. The amplification stage is divided into two levels made with sustainable and discrete components. In addition, a variable resistance makes it possible to adjust the gain. Finally the output circuit adjusts the impedance equal to $50 \Omega$.

Moreover, the amplifier has two different outputs:

- The first one, called differentiated output, is connected to the input of the discriminator. It provides shorts signals so as the electronic supports high count rate (The count rate can reach $6.10^{6} \mathrm{cps}$ for fission chambers and 2.9.10 $\mathrm{cps}$ for proton recoil detectors without saturation).

- $\quad$ The second one, called not differentiated output, is generally plugged with $50 \Omega$ resistance. It provides signals which shape is adapted to spectroscopy 
amplifier, so that it can be used to determine the threshold value of the discriminator (interesting events corresponding to fast neutrons interaction in the detector are selected by their higher amplitude)

\section{B. Gain analysis}

The gain is defined as in Eq. 1:

$$
G=\frac{V_{\text {out }, \max }}{V_{\text {in, } \max }} \quad \frac{[V]}{[V]}
$$

As the prototypes characteristics are identical compared to the old electronics, the new amplifier gain was measured in order to set a similar value. It was decided to set the gain to 910 as resumed in Table III.1.

In addition, it is interesting to study the evolution of the signal/noise ratio according to the gain. As shown in Figure III.1, the value is constant for each measured gain.

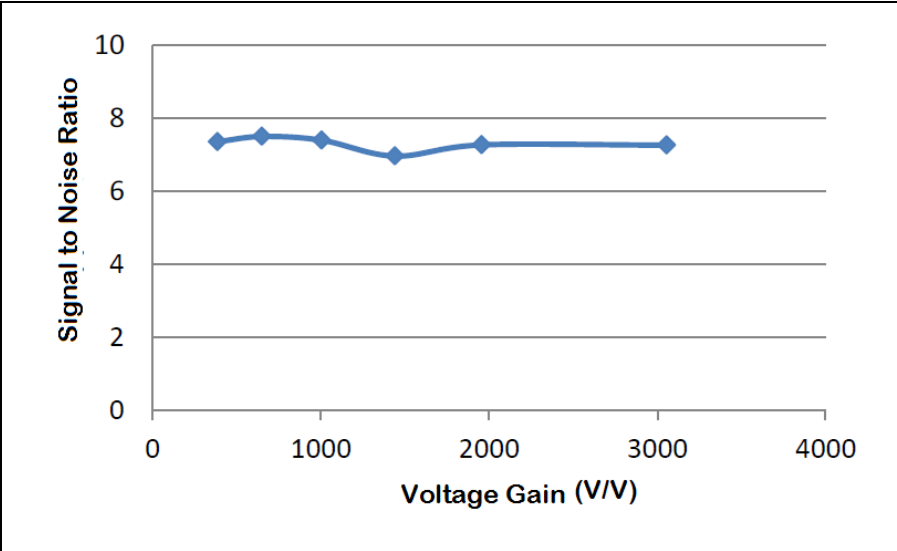

Figure III.1 - Voltage Gain vs Signal/Noise ratio for the Prototype

TABLE III.1 - MEASURED GAIN

\section{Original $(\mathrm{V} / \mathrm{V})$}

1020

Prototype (V/V)

910

\section{Amplifier Noise}

In this section, the noise of the amplifier obtained during the measurement campaign is analyzed.

Figure III.2 and Figure III.3 show the noise level of the original and the new amplifier, respectively. The noise level of the new amplifier is slightly smaller than the old one. This result is probably linked to the lower gain of the prototypal amplifier compared with the original amplifier (cf. Table III.1).

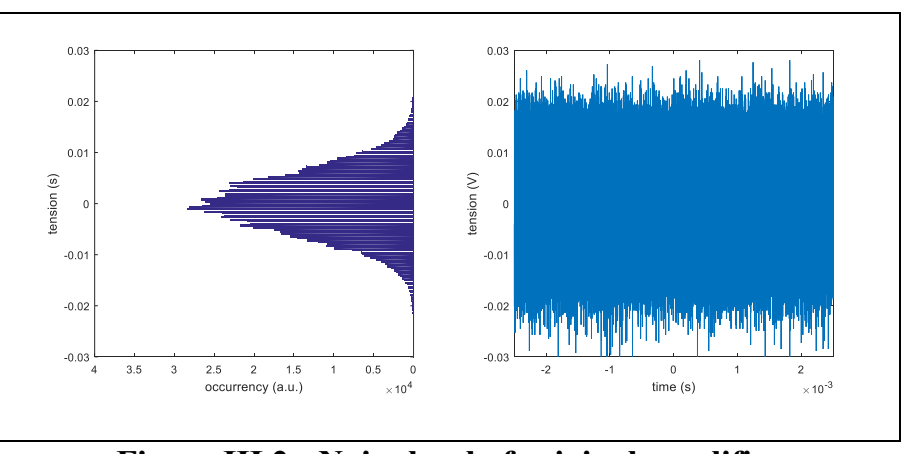

Figure III.2 - Noise level of original amplifier

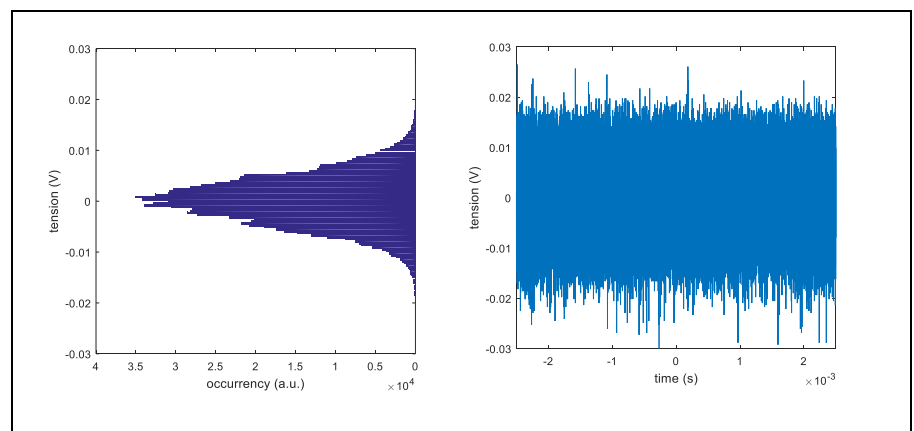

Figure III.3 - Noise level of new prototype amplifier

\section{Amplifier Response}

In this section the response of the amplifier tested under different conditions is discussed. First of all, the amplifier was tested using a calibrated function generator. Thereafter, its response to an Am-Be source was observed.

\section{1) With function generator}

In this part, the response of the original amplifier compared to the old amplifier is shown. The measurement was performed using a generator function. The input waveform has the characteristics listed in Table III.2.

\section{TABLE III. 2 - GENERATED IMPULSIONS}

Full Weight at Half

Maximum (FWHM)

$40 \pm 2 \mathrm{~ns}$

Min Value

Frequency

$$
-3.7 \pm 1 \mu \mathrm{A}
$$

$5 \mathrm{kHz}$

These impulsions were injected into the amplifier. In Figure III.4 and in Table III.3, measured characteristics of the pulses delivered by the differentiated output are shown. It is possible to see that the new prototype produce a waveform smaller than the original amplifier. This is due to different gain of two amplifiers. However, shapes of both waveforms are quite similar. All the impulsions are correctly amplified. 
TABLE III. 3 - WAVEFORM CHARACTERISTICS FOR DIFFERENTIATED OUTPUT

\begin{tabular}{l|l|l}
\hline \hline & Original & Prototype \\
\hline FWHM & $28 \pm 2 \mathrm{~ns}$ & $38 \pm 2 \mathrm{~ns}$ \\
Min Value & $-194 \pm 5 \mathrm{mV}$ & $-170 \pm 5 \mathrm{mV}$ \\
Frequency & $5 \mathrm{kHz}$ & $5 \mathrm{kHz}$
\end{tabular}

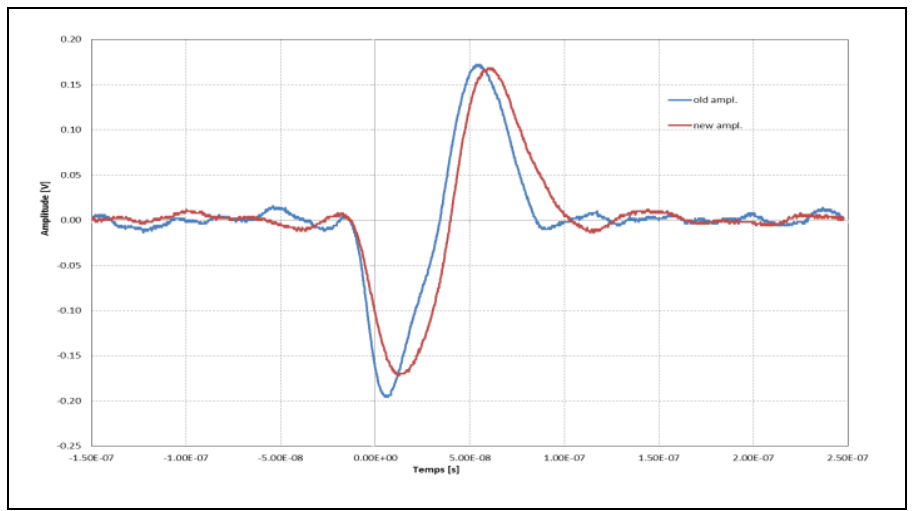

Figure III.4 - Waveform for differentiated output

\section{2) With Am-Be Source}

A test of the entire measurement chain was realized with an Am-Be source placed in front of a fission chamber. The original amplifier/discriminator and the new one have been permutated in order to observe the response of the electronic chain without any detectors influence. The source used for this test emits $4.3310^{5} \mathrm{n} / \mathrm{s}$ over $4 \pi$ solid angle. This source produces very small neutron flux compared to neutronic current coming from CABRI core $\left(\sim 10^{9} \mathrm{n} \cdot \mathrm{cm}^{-2} \cdot \mathrm{s}^{-1}\right)$.

Figure III.5-a shows a histogram (including 1000 impulsions) of the pulses amplitudes, measured with a digital oscilloscope, for the differentiated output of the old amplifier. Otherwise, the same distribution calculated for the new prototype is shown in Figure III.6-a.

In Figure III.5-b, the distribution of FWHM of pulses delivered by the original amplifier (differentiated output, negative part) is shown. This graph allows us to appreciate the stochastic behavior of the detector chain. This distribution could be assimilated to a Gaussian distribution centered on 21 ns with a standard deviation of 1.6 ns. In Figure III.6-b, the same analysis was carried out for the new prototype. This distribution is centered on $24 \mathrm{~ns}$ with a deviation of $1.3 \mathrm{~ns}$.

As shown in figures, both distributions are similar. Hence it was concluded that the behavior of the new amplifier fulfills all requirements for CABRI experiments.

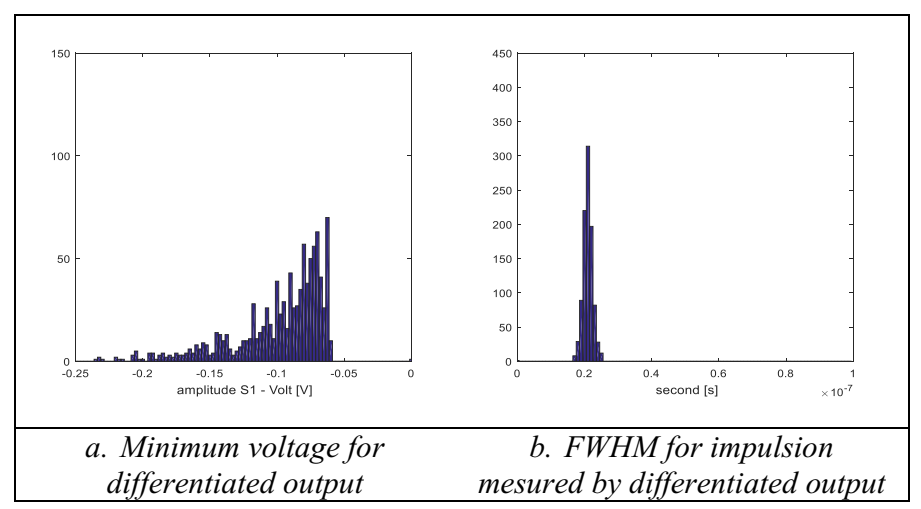

Figure III.5 - Characterization of original amplifier with Am-Be source

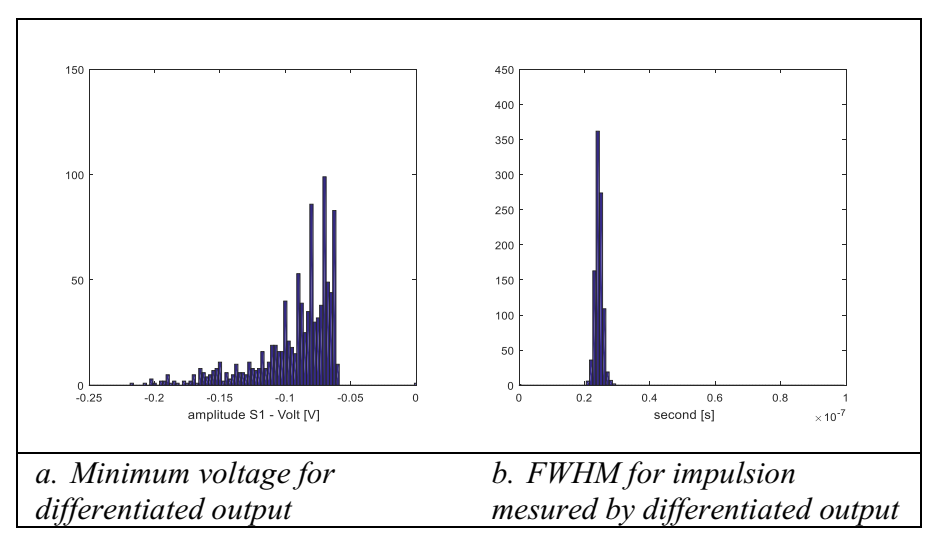

Figure III.6 - Characterization of new prototype amplifier with Am-Be source

Finally, the Table III.4 presents the count rates of pulses delivered by the differentiated output of both old and new amplifiers. These results are consistent with experimental conditions.

TABLE III.4 - COUNT RATE FOR AM-BE SOURCE AFTER ONE HOUR EXPOSURE

\begin{tabular}{l|c|c|c}
\hline \hline & Gain $(\mathrm{V} / \mathrm{V})$ & $\begin{array}{c}\text { Discriminator } \\
\text { Threshold }(\mathrm{mV})\end{array}$ & $\begin{array}{c}\text { Count Rate } \\
(\mathrm{cps})\end{array}$ \\
\hline Original & 1020 & $-41.8 \pm 0.5$ & $0.30 \pm 0.01$ \\
Prototype & 910 & $-33.0 \pm 0.5$ & $0.39 \pm 0.01$
\end{tabular}

\section{DISCRIMINATOR}

\section{A. Description}

The discriminator is connected just downwards the amplifier. This component is used to create a TTL signal whenever the minimum value of the impulsion (negative part), which comes from the amplifier, is lower than a given threshold.

These TTL signals are counted by the computer.

The discriminator consists of 4 stages:

- The input stage, used to create the input voltage of the comparator, and to generate the threshold voltage as described by Eq. 2; 
- The comparator stage, which compares the minimum value of the signal produced by the amplifier with a threshold;

- The shaping stage, which makes TTL signals when stimulated by the comparator stage

- The output stage, which is realized to charge the discriminator with $50 \Omega$ impedance.

The threshold signal is constructed by two components, so that it may be changed either by means of a potentiometer or by remotely by computer. In fact, the computer produces an analogical voltage, by the means of a Digital/Analogical board, $\left(\mathrm{V}_{\text {ext }}\right)$ which is injected at the entrance of the discriminator and added to the potentiometer threshold $\left(\mathrm{V}_{\mathrm{p}}\right.$, given in potentiometer turns; 10 potentiometer turns are equal to $-5 \mathrm{~V})$. With this system, the discriminator response can be easily determined as a function of the threshold.

$$
V_{s}=\frac{V_{p}}{-2}-V_{e x t} \quad[m V]
$$

Eq. 2

\section{B. TTL signal produced}

When the shaped impulsions pass the threshold, a standard TTL signal is produced by the discriminator. TTL signals allow transporting easily information by the means of a normal coaxial cable to the computer. To be registered by the computer, the amplitude and the width of signals delivered by the discriminator should be greater than $1.5 \mathrm{~V}$ and $5 \mathrm{~ns}$, respectively.

Figure IV.1 shows the shape of the two different TTL signals produced by the original discriminator and the prototype.

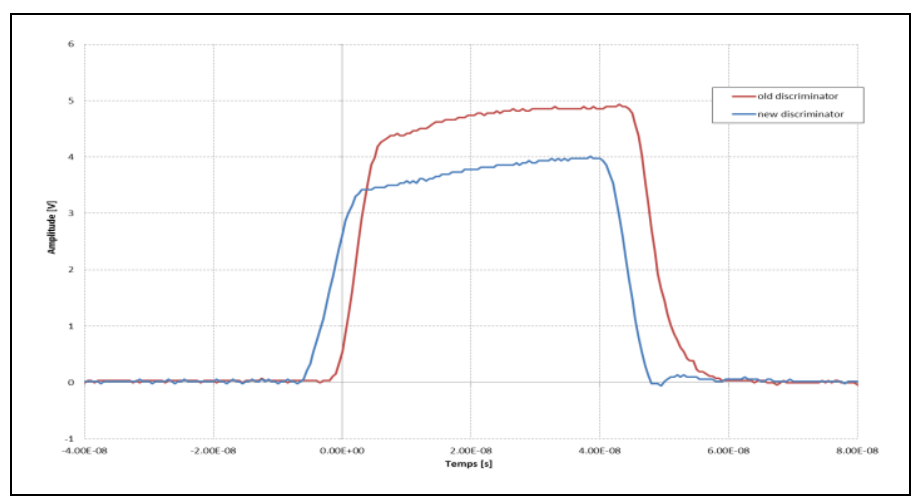

Figure IV.1 - TTL signal of the original discriminator compared to new prototype

In Table IV.1 the characteristic of the TTL signals produced from the new discriminator and the original one are presented. Characteristic of input signals are given in Table III.2.

The characteristics of the new discriminator are consistent with the original one.

TABLE IV.1 - TTL CHARACTERISTICS

\begin{tabular}{l|cc}
\hline \hline \multicolumn{2}{c}{ Original } & Prototype \\
\hline FWHM & $46 \mathrm{~ns}$ & $47 \mathrm{~ns}$ \\
\hline
\end{tabular}

\begin{tabular}{l|ll}
\hline Height & $4.5 \mathrm{~V}$ & $3.5 \mathrm{~V}$ \\
\hline Frequency & $5 \mathrm{kHz}$ & $5 \mathrm{kHz}$
\end{tabular}

\section{ANALYSIS WITH CABRI CORE}

\section{A. Discriminator threshold determination}

The determination of the threshold value for the discriminator is quite important in order to realize measurements with $\mathrm{FC}$ or PR.

The threshold has to be adjusted so as the discriminator delivers TTL signals whenever fast neutrons (interested events) either emitted by the CABRI core or the test rod interact in the detector. The threshold aims at suppressing pulses coming from noise. The noise can be produced either by electronic and or not interesting particles interacting in the detector (gamma, alpha for fission chamber). The discriminator threshold has to be adjusted whenever electronics or detectors characteristics change.

The electronic chain response has been determined according to threshold values (cf. Eq. 2), changing with $5 \mathrm{mV}$ steps.

In Figure V.1 the response can be observed: new prototype has exactly the same behavior compared to the original one.

Furthermore this figure shows that the threshold is chosen (cf. TABLE III.4) for the two electronic chains so as to eliminate noise leading up to excessive counting. Only interesting events in the plateau zone are registered. Thresholds have not been modified.

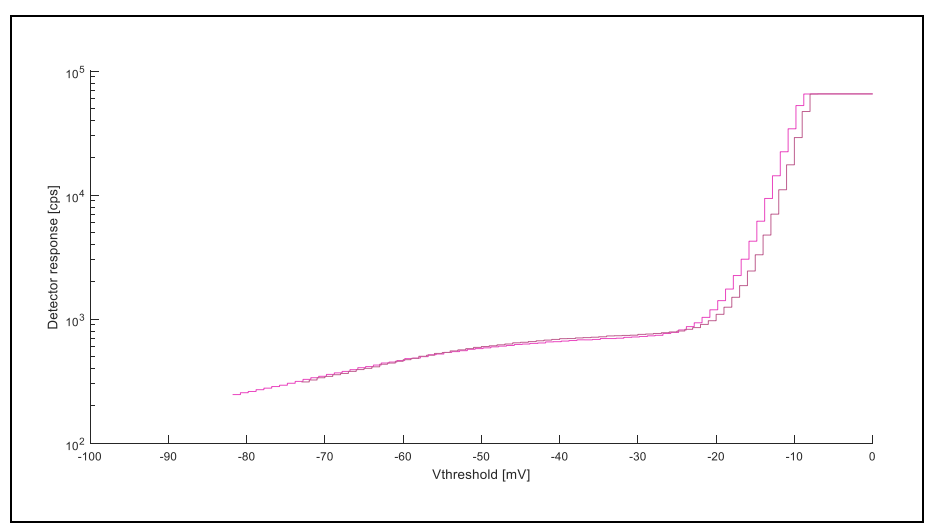

\section{Figure V.1 - Detectors response vs. Discriminator threshold (in fuchsia the new prototype, in violet the original one)}

\section{B. Chain dead time evaluation}

Another aspect of this new prototype concerns the dead time of the chain. In order to control properly the dead time of the chain, the constructor has imposed the dead time of the chain using a great dead time into the discriminator to produce the TTL signals. In this way, the dead time of the chain is constant, and does not depend anymore neither on collection time of the couples electron/ion at the detectors electrodes, nor on the propagation time of pulses throw the amplifier.

Using this concept, Eq. 3 can be written: 


$$
\tau_{\text {chaine }}=\tau_{\text {det }}+\tau_{\text {amp }}+\tau_{\text {discr }} \cong \tau_{\text {discr }}
$$

Eq. 3

With $\tau_{\text {det }}, \tau_{\mathrm{amp}}, \tau_{\mathrm{discr}}$ the dead time generated by the detector, the amplifier and the discriminator, respectively.

Therefore, we can measure it without using neutron source.

A function generator with two channels is used to measure the dead time [2]. It is set so that the first channel generates, square pulse train with frequency $\mathrm{f}_{1}$, and the second channel generates square pulse train with frequency $f_{2}$. The two pulses trains are injected at the amplifier input, and the TTL signals are viewed by means of an oscilloscope at the discriminator output. At the beginning, $f_{1}$ is equals to $f_{2}$ and the count rate amounts to $f_{1}+f_{2}$. Changing the frequency of the second pulse train, the second TTL signal observed at the discriminator output get close to the first, until it disappears (count rate equals to $\mathrm{f}_{1}$ ). The time measured between the two pulses just before the second one disappears corresponds to the dead time. Using this methodology, dead times, presented in Table V.1, are evaluated.

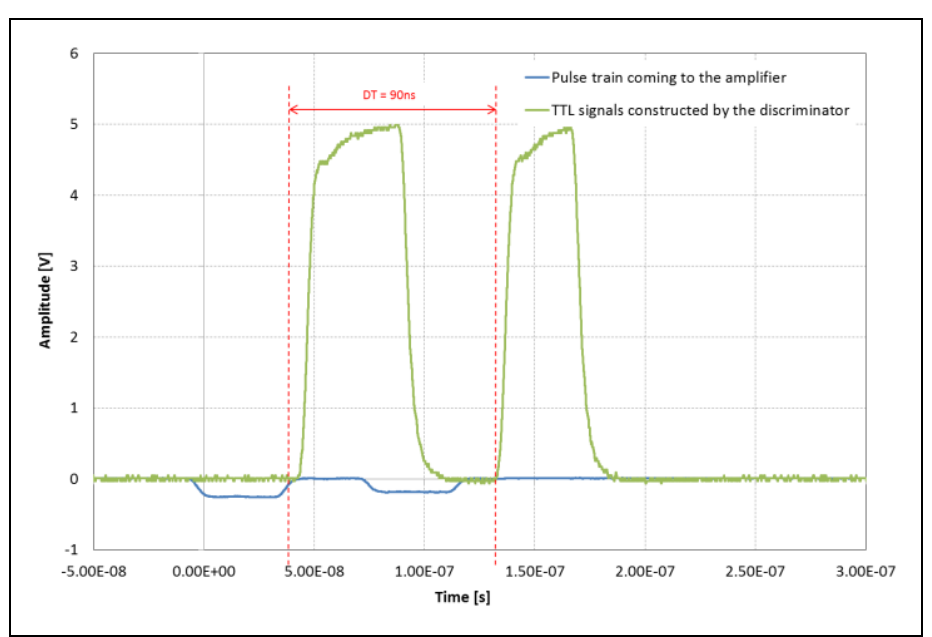

Figure V.2 - Two different pulse trains just before losing the second one

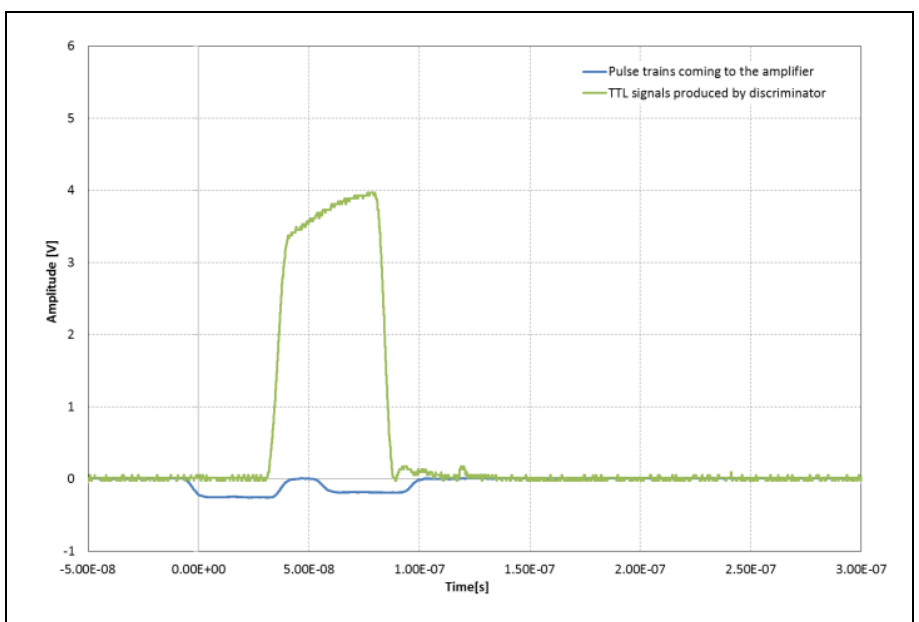

Figure V.3 - Single pulse train just after losing the second one
TABLE V.1 - DEAD TIME

\begin{tabular}{cc}
\hline \hline Original & New prototype \\
\hline $90 \mathrm{~ns}$ & $88 \mathrm{~ns}$
\end{tabular}

These results are consistent, the new electronic works properly. These values are used in parallelizable dead time formula [3] which gives us the real count rate.

\section{Detector linearity}

In this section, the linear responses of original and prototype chains have been analyzed. Comparison is made using a boron deposition chamber placed near the core [4].

This boron detector is stimulated by thermal neutron coming from the core, both in steady state conditions (power plateau) and in transitory condition (during power peak).

Our ${ }^{237} \mathrm{~Np}$ detectors are sensible to fast neutrons. In any case, the proportion of thermal neutron and fast neutron is constant in steady state condition. It is assumed that this ratio is constant also during transient of the reactor [5].

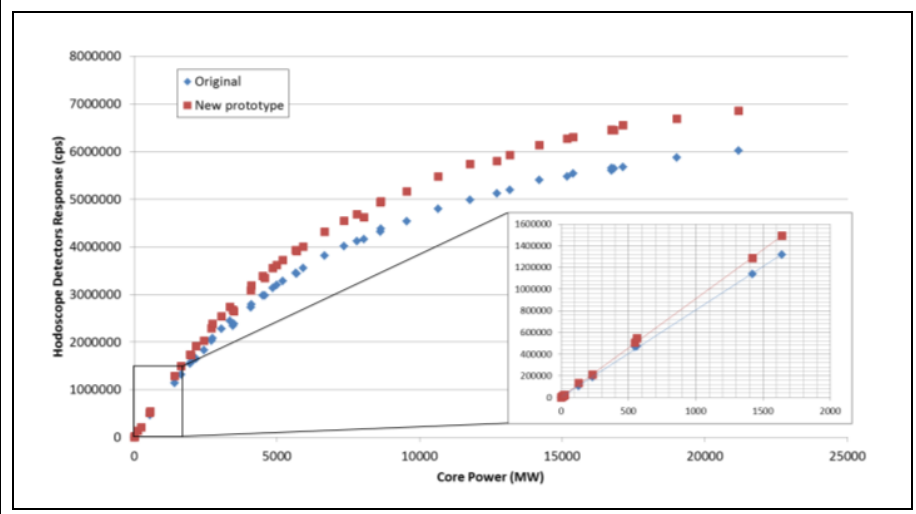

Figure V.4 - Detectors responses at different powers of CABRI reactor without dead time correction

In Figure V.4 the count rates of original and new prototype electronics chain vs core power measured by boron detectors is shown. From this figure it is possible to see that the counting chains including fission chambers have a linear behavior under 1.5 million cps which corresponds to $\sim 1.6 \mathrm{GW}$ of CABRI power. For higher power, a dead time correction has to be taken into account, due to the behavior of the electronic chain [3].

Two chains do not give the same response because they are connected to different FC. If we normalize signals to the maximum values of each set, the two curves will superpose perfectly.

\section{CONCLUSION}

All tests realized in this campaign fulfill our expectation. The response of the new prototype is similar to its predecessor respecting all needed requirements for our experiments. Furthermore, this new solution will be easier to maintain thanks to the full discrete architecture of the amplifier. These 
new devices will be installed in CABRI Hodoscope measurement system current 2018.

In the near future, an industrialization campaign will be programmed in order to supply more devices for others measurement chains of the hodoscope.

\section{REFERENCES}

[1] G. Baratte, «Ensemble de mesure pour hodoscope,» CEA LIST, Saclay.

[2] J. Gostely et E. Carnal, «La methode des deux oscillateurs pour la mesure du temps mort dans l'instrumentation nucléaire,» Nuclear instruments and methods, 1978.

[3] V. Chevalier, S. Mirotta, J. Guillot and B. Biard, "The CABRI fast neutron Hodoscope: renovation, qualification program and first resutls following the experimental reactor restart," IRSN, Cadarache, 2017.

[4] J. Lecerf, "Study of the linearity of CABRI experimental chambers during RIA transients," CEA, Cadarache, 2017.

[5] G. Augier and K. Baumung, "Quantitative fuel motion determination with the CABRI fast neutron hodoscope : evaluation methods and results," Nuclear Technology, 1990. 